\title{
AUTOBIOGRAPHISCHES SCHREIBEN IN ALFRED ANDERSCHS PROSA DER 50ER JAHRE
}

\author{
ABSTRACT. Autobiographical Writing in the Alfred Andersch's Prose from the 1950s
}

Alfred Andersch's autobiographical texts from the 1950s have been heavily criticized in recent literature on the topic. W.G. Sebald's essay about Andersch was of crucial importance. The details of Andersch's stay in the Dachau concentration camp as well as the writer's motivation to desert at the end of the war were questioned. The article aims at a new reading of Andersch's autobiographical texts with regard to their credibility. It compares the early short story Flucht in Eturien with the autobiography Die Kirschen der Freiheit and a few less known texts. The analysis leads to the conclusion that Andersch "re-wrote" his biography as a creation that fulfils unconscious wishes of a whole generation. His intention was to adapt the image of decent young men of antifascist beliefs whose only guilt was the loyalty to their comrades.

KEY WORDS: Alfred Andersch, autobiographism, desertion, the issue of guilt, post-war literature

\section{1.}

Alfred Andersch, einer der bekanntesten Vertreter der Nachkriegsgeneration, ging vor allem als Mitbegründer der Gruppe 47 in die Literaturgeschichte ein. Er war nicht nur Autor von Romanen und Erzählungen, sondern arbeitete auch als Zeitungs- und Radiojournalist und war darüber hinaus ein leidenschaftlicher Reisender. Er berichtete über verschiedene Länder, die er besuchte, beschrieb Landschaften vom Nordpol bis Mexiko sowie diejenigen von Großbritannien, Irland und Skandinavien, Frankreich, Spanien, Portugal, Italien. ${ }^{1}$ Am meisten fühlte er sich der italienischen Kultur verpflichtet (Jabłkowska, 2016), weil ihn mit Italien Erlebnisse verbanden, die auf sein Leben einen tiefen Einfluss hatten. Eine persönliche, subjektive Reflexion und ein Bezug zur eigenen Biographie, über die sich Andersch sehr offen

\footnotetext{
${ }^{1}$ Die bekanntesten Reisebücher Anderschs sind Hohe Breitengrade oder Nachrichten von der Grenze (1969) und Norden Süden rechts und links: von Reisen und Büchern 1951-1971 (1972) (Kunz, 1994; Bargelli, 2008).
} 
äußerte, sind charakteristisch für sein ganzes Schaffen. In einer späten, erst 1976 verfassten Erinnerungsskizze mit dem Arbeitstitel Der Seesack nennt er einige seiner Werke, die autobiographische Züge tragen:

(...) Festschrift für Captain Fleischer und Mein Verschwinden in der Providence (...) [das] Gedicht Erinnerung an eine Utopie. Auch habe ich zu den Kirschen Nachträge geliefert: die Erzählungen Alte Peripherie, Die Inseln unter dem Winde und Brüder; sie handeln, wie übrigens auch jene Erinnerung an einen amerikanisch-jüdischen Militärarzt namens Fleischer, von einem gewissen Franz Kien, der ich selber bin. (Andersch, 2004b, S. 424)

Doch die wichtigsten autobiographischen Texte entstanden in den 1950er Jahren, als der Autor sich erst als Dichter zu entwickeln begann. In dieser Zeit schrieb er Die Kirschen der Freiheit, einen Bericht über seine Jugend in München, die kommunistischen Anfänge und schließlich die Fahnenflucht, die ihm in Italien gelang. Dies waren prägende Erlebnisse, die zur Entwicklung des später engagierten, politisch interessierten Schriftstellers beitrugen und ihm Impulse für weitere Arbeiten gaben. Mit Die Kirschen der Freiheit ist er als Schriftsteller bekannt geworden, und bis zu seinem Lebensende beteuerte er die ,Wahrheit' und den bekenntnishaften Charakter des Berichts. Wie stark Andersch auf dem autobiographischen, faktualen Charakter von Die Kirschen der Freiheit beharrte, schreibt Döring, 2011 (bes. S. 13 und folgende, auch Alfred Andersch im Gespräch mit Jürg Acklin, 1992). Der Herausgeber von Anderschs Gesammelten Werken, Dieter Lamping, attestiert den Aufzeichnungen eine Authentizität, an der „kein Zweifel“ bestehe (Lamping 2000, S. 219). Angesichts der Debatte, die nach W. G. Sebalds Publikationen zu Anderschs NS-Vergangenheit begann (Sebald, 1999; auch Sebald, 1993), sowie der Zweifel am Wahrheitsgehalt des autobiographischen Berichts, der in der Forschungsliteratur angedeutet wurde (Reinhardt, 1990; Tuchel, 2008), recherchierte Döring sehr gewissenhaft im Marbacher Literaturarchiv, wo sich der Nachlass von Andersch befindet. Anliegen war, das Manuskript von Die Kirschen der Freiheit zu untersuchen und aus seiner Entstehung, aus den Randnotizen, Modifizierungen, Streichungen etc. „Rückschlüsse auf bestimmte Literarisierungsnotwendigkeiten“ (Döring, 2011, S. 17-18), also auf Stellen zu ziehen, die der Autor „kontrollieren“ wollte, die ihm also selbst „besonders problematisch“ erschienen (Döring, 2011, S. 18). Das Ergebnis ist,

\footnotetext{
dass Andersch [den] heiklen Teil seiner Biographie in der Endfassung seines autobiographischen Berichtes Die Kirschen der Freiheit nicht etwa nur verdrängte, sondern offenbar bewusst aussparte. Nur weil Sebald (...) den Gang ins Archiv scheute, blieb ihm ein zentraler Beleg seiner Argumentation unbekannt. (...) Sebald wußte gar nicht, wie recht er hatte. (Döring, 2011, S. 40)
}

Ähnliches konstatiert auch Rolf Seubert in demselben, von Döring und Manfred Joch herausgegebenen Band. Er bespricht Dokumente zu Anderschs KZ-Haft und weist viele Ungenauigkeiten nach, die dem Schriftsteller bewusst sein mussten (Seubert, 2011). Es bewährt sich also, was Johann Tuchel bereits einige Jahre zuvor 
riet, nämlich vorsichtig mit autobiographischen Schriften von Alfred Andersch umzugehen (Tuchel, 2008). Seubert rekonstruiert akribisch die Ereignisse, die im ersten Teil der Kirschen der Freiheit genannt werden. Es handelt sich vor allem um die Verhaftung im März 1933, die Haft in Stadelheim und die Einlieferung in Dachau. Seubert konstatiert nach einer sehr sorgfältigen Recherche, dass Anderschs Beschreibung der Dachauer Haft nicht nur ungenau und oberflächlich sei - was die Forschungsliteratur bereits feststellte -, sondern dass diese Beschreibung an mehreren Stellen mit der historischen Realität nicht übereinstimmt. Außer kleineren Details entspricht insbesondere die Schilderung des Schlüsselerlebnisses, der Ermordung von zwei jüdischen Häftlingen, die bei Andersch „Goldstein“ und „Binswanger“ heißen - in Wirklichkeit waren es drei Häftlinge: Ernst Goldmann, Rudolf Benario und Erwin Kahn - nicht den Tatsachen (Seubert, 2011, S. 81-123). Ähnlich ungenau, geradezu fahrlässig, sei - so Seubert - die Erinnerung an Wilhelm Franz: „Der lange, knochige, eisenharte Willi Franz, berühmter Bergsteiger, hatte sich damals noch nicht erhängt, aber er spielte bereits so schlecht Schach, daß ich ihn mit Leichtigkeit schlug" (Andersch, 1968, S. 41). Seubert weist nach, dass Wilhelm Franz sich nicht erhängt hat, sondern erhängt wurde. Andersch zeige mit der kurzen Schachepisode ,wenig Anteilnahme am wirklichen Schicksal des ehemaligen Freundes" (Seubert, 2011, S. 136). Somit könne man die Authentizität von Anderschs Dachauer Erinnerungen in Frage stellen. Dies sind nur zwei Beispiele aus dem hundert Seiten umfassenden Artikel, der schließlich zum grundsätzlichen Zweifel an Anderschs Haft in Dachau führt:

Es sieht so aus, als habe die Erzählung seiner KZ-Haft und der angeblichen Verfolgung, während der gesamten NS-Zeit vor allem dazu gedient, die spätere Desertion aus der Wehrmacht mitsamt der Begründung als Konsequenz echter , antifaschistischer' Haltung erscheinen lassen. (...)

Anderschs substanzarme Schilderung des Dachauer Häftlingsalltags [stimmt] so gut wie nicht mit den zahlreichen Zeugenaussagen überein (...). Und auch seine zeitliche Überdehnung der Haft auf drei bis sechs Monate legt nahe, dass es ihm auch um die Schaffung und Pflege einer Opferbiographie zu tun gewesen sein muss. (Seubert, 2011, S. 138-139)

Dies bestätigt auch Johannes Tuchel in einem kurzen Nachtrag zu Seuberts Artikel, in dem er zwar an einigen Stellen das harte Urteil des Historikerkollegen abschwächt, doch insgesamt zugibt, „Seuberts Studie“ bestätige den Befund, dass sich Alfred Andersch in seinen „Äußerungen (...) nach 1945 wie viele andere seiner Zeitgenossinnen und Zeitgenossen neu erschaffen hat" (Tuchel, 2011, S. 147).

Die Historiker vergessen allerdings, dass eine Autobiographie kein Dokument ist. Auch wenn der Autor oder die Autorin vom Wahrheitsgehalt der eigenen Bekenntnisse überzeugt sein mag, sind Texte, die persönliche Erinnerungen enthalten, „Dichtung und Wahrheit" - Goethe hat dies sehr knapp formuliert, lange bevor die einschlägigen Studien zum Thema Erinnerung und Gedächtnis verfasst wurden. Auch aufgrund der zur Verfügung stehenden Dokumente lassen sich viele Einzelheiten aus Alfred Anderschs Leben in der Zeit des Nationalsozialismus nicht mehr 
rekonstruieren. Der Autor erzählte über sich selbst und ,erschrieb“ sich seine Biographie zwar neu, wir können jedoch nicht behaupten, er habe sich an die ,Wahrheit erinnern können, wollte sie aber verheimlichen oder er habe ,gelogen'. Es ist möglich, dass unbewusste Verdrängungsstrategien verursachten, dass die kommunistische Episode sowie die Verhaftung Anfang der 1930er Jahre von Anderschs Gedächtnis etwas ,ausgebessert' wurden - möglicherweise war er nicht in Dachau inhaftiert; dass er aber verhaftet war, haben Historiker doch nachweisen können. Nicht ohne Bedeutung ist hierbei die positive Rezeption der Die Kirschen der Freiheit. Zahlreiche Zeitungsbesprechungen, die nach der Publikation des Buches erschienen, zeugen vom Bedürfnis der damaligen Leser (wahrscheinlich vor allem männlicher Leser), ihre eigene Soldatenbiographie, das Schicksal ihrer Generation als Fehltritt zu interpretieren, der sich gleichwohl intellektuell erklären und bewältigen lassen könnte. Die vierzig Jahre nach Erscheinen des Buches zusammengestellten Besprechungen (Stephan, 1992) attestieren Andersch Außergewöhnlichkeit, Offenheit, Ehrlichkeit, Klarheit, Willensfreiheit. Heinrich Böll nannte Anderschs Buch eine „Wohltat“ (Böll, 1992, S. 133). Nichtsdestoweniger wird nicht selten der Vorwurf des Idealismus, der Introvertiertheit, der Subjektivität, des Anarchismus oder einer politischen Sentimentalität formuliert. Oft wird das Werk mit dem fast zeitgleich erschienenen Waldgang (1951) von Ernst Jünger verglichen, meist jedoch zuungunsten Anderschs. Hans Egon Holthusen betonte in einer relativ sehr umfangreichen Rezension die Bedeutung von Anderschs Buch, das typisch für die „Intellektuellen der sogenannten heimatlosen Linken" sei (Holthusen, 1992, S. 126). Gleichzeitig sieht er auch Schwächen in Die Kirschen der Freiheit: die Übertragung des Sartreschen Freiheitsbegriffes auf die aktuelle Politik (Holthusen, 1992, S. 122), eine utopische Protesthaltung, die sich gegen jede politische Realität wendet, doch nicht produktiv sei (Holthusen, 1992, S. 120), schließlich auch die Überbewertung der eigenen Tat:

Wenn Andersch sagt, seine Desertion sei sein „ganz kleiner privater 20. Juli“ gewesen, so ist das sicherlich irreführend. Es ist zweierlei, ob man sich mit dem Fahrrad seitwärts in die Büsche schlägt, oder ob man mit Sprengstoff in den Sperrkreis Eins des Führerhauptquartiers eindringt, um das Ungeheuer zu erledigen, und dann, unter unvorstellbaren Qualen, seine Tat mit dem Leben bezahlt. (Holthusen, 1992, S. 124)

Doch auch wenn sie an Anderschs Idealismus oder auch Stilisierung zum Widerstandskämpfer Kritik übten, begrüßten die meisten Rezensionen den antifaschistischen Gestus in Die Kirschen der Freiheit und die Hinwendung zu neuen Werten.

2.

Für die Literaturwissenschaft, der die beiden zuvor genannten Historiker Ungenauigkeiten, ja Fahrlässigkeit und Naivität vorzuwerfen scheinen, ist die biographische ,Wahrheit` zwar wichtig, wichtiger ist indessen, welche Funktion die Verdrän- 
gungsmechanismen hatten und wie Andersch seine Biographie literaturästhetisch ausrichtete. Jörg Döring hat recht, wenn er behauptet, die Aussparung von manchen Episoden, Streichungen oder Hinzufügungen ließen den privaten Teil der Biographie außen vor ,zugunsten einer stärkeren Konzentration auf die Deserteursthematik“ (Döring, 2011, S. 43). Und in diesem Fall bemühte sich Andersch nicht nur, das eigene Selbstbild zu stärken oder sich selbst als antifaschistischen jungen Mann zu präsentieren. Deutlich trat er auch als Vertreter seiner Generation und zudem seiner sozialen Schicht auf und rückte sich so eine kollektive Identität zurecht. Auch wenn diese Identität nicht ganz historisch dokumentiert werden konnte, stimmt sie mit dem überein, was er als Autor nach dem Zweiten Weltkrieg vertreten wollte. Dies war der ,autobiographische Pakt“ (nach Lejeune, 1994), den er mit dem Leser einging, und in diesem Sinn war sein Bericht über die Desertion und Flucht, authentisch".

Alle Autoren dieser Jahrgänge waren von der Jugend oder Kindheit in der Weimarer Republik und im Nationalsozialismus sowie von der (im Falle eines entsprechenden Alters hinzukommenden) Militärzeit stark beeinflusst. Anderschs Offenheit, mit der er über seinen frühen Lebensweg schrieb, mag auf der Überzeugung gebaut haben, dass er als ehemaliger KZ-Häftling mit kommunistischen Überzeugungen und dann Deserteur eher Opfer des Nationalsozialismus als Täter sei. Seine Autobiographie, die er 1950, 1951 verfasste, bestätigt diese Selbstvorstellung, verrät allerdings auch Verfänglichkeiten, die die Selbstkontrolle noch nicht korrigierte. Die Forschungsliteratur befasste sich nach dem Tod des Autors, nicht zuletzt wegen der von Sebald provozierten Kontroverse, eingehend mit Anderschs Biographie, und es wird schwierig sein, dem etwas an konkreten Fakten hinzuzufügen. Ich versuche dennoch, Die Kirschen der Freiheit noch einmal ,hermeneutisch` zu lesen - auch wenn dies nicht vollends gelingen mag, denn das historische Wissen drängt sich von selbst auf.

Aus großer zeitlicher Distanz schreibt Klaus R. Scherpe 2015 über Anderschs politisch orientierte Werke der unmittelbaren Nachkriegszeit, dass sie sich die „Sprache der anarchischen Revolte“ angeeignet haben, die „nicht frei vom Weltanschauungsjargon der 1920er und 30er Jahre" sei (Scherpe, 2015, S. 441). Andersch versuchte - sich auf das Vorbild Ernst Jüngers beziehend - die Wirklichkeit in „minutiösen Mitteilungen (...) verdichtet und zugleich intensiviert“ (ebd., S. 442) in der Sprache festzuhalten:

Die im Erlebnisbericht der Kirschen der Freiheit (...) zum ersten Mal erprobte Stilisierung exponiert (...) ,Augenblickszustände ' oder ,Kristallisationspunkte‘. (....) Die Inszenierungen der ,Augenblickszustände“ in den Kirschen der Freiheit sind nicht historisch oder politisch in ihrem realen Erlebnisgehalt (...), vielmehr präsent in dem, was er als Eklat sprachlich in Szene setzt. (ebd.)

Auf diese Weise gelingt es Andersch, sein Leben - das Leben eines jungen Mannes im Nationalsozialismus - als eine durchaus ,normale' Entwicklung zu beschreiben, die sehr entfernt von jeglicher Mitschuld und Mitverantwortung am 
damaligen politischen Geschehen war. Die erste Erinnerung an die Kindheit, die er festhält, betrifft die Ereignisse nach der Niederlage der Münchner Räterepublik. Sein national gesinnter Vater war mit der Todesstrafe für die Revolutionäre einverstanden. Als fünfjähriger Junge begriff Alfred noch wenig von dem, was er sah. Zehn Jahre später interessierte den Fünfzehnjährigen - so der autobiographische Bericht, der von einem ca. Siebenunddreißigjährigen geschrieben wurde -, warum die Mitglieder des Erschießungskommandos die Verurteilten nicht laufen ließen. Andersch zeigt sich selbst als pazifistischen jungen Mann, der sich im Widerspruch zu den Erziehungsmaßnahmen des Vaters entwickelte. Im Kapitel Verschüttetes Bier erzählt er von seinem Engagement in der kommunistischen Bewegung. Er überbewertet seine Rolle nicht, er ist sich bewusst, dass er glimpflich davongekommen ist: „Mein lumpiges Vierteljahr Haft zählt nicht gegen die zwölf Jahre, die viele meiner Genossen hinter dem Draht von Lagern verbrachten" (Andersch, 1968 [Erstausgabe 1952], S. 39-40). Der Bericht über die Jahre 1932 und 1933 erweckt gleichwohl den Eindruck, dass über den Sieg des Nationalsozialismus einige Zufälle entschieden hätten und der politische Wille der Menschen ganz anders orientiert gewesen sei; dem ungeachtet habe niemand genügende Entscheidungskraft gezeigt, und so hätten die Nazis die Macht erobert. Auch Klaus R. Scherpe weist auf die Schilderung der Ereignisse vom März 1933 hin, als „,das Gewerkschaftshaus von der SA besetzt wurde" (Andersch, 1968, S. 34). Ein SA-Mann hatte einen kleinen Unfall mit seinem Motorrad, und die schweigend stehenden und zuschauenden Arbeiter hätten in diesem Moment die Möglichkeit gehabt, gegen die neuen Machthaber zu protestieren:

Dies wäre der Augenblick des Aufstands gewesen, der Deutschland vielleicht ein anderes Gesicht gegeben hätte. (...) Sicherlich, es wäre nur ein kleiner Sieg gewesen, eine rasch verwehende Tat, morgen ausgelöscht im Orkan der Niederlage - aber es hätte genügt, hätte den Staatsstreich in ein für alle sichtbares Blutbad verwandelt und den Schein der ,Ordnung' zerstört. Aber ich stieß den Schrei nicht aus. Niemand. (Andersch, 1968, S. 35; vgl. dazu Scherpe, 2015, S. 443)

Andersch versucht offensichtlich den Eindruck zu erwecken, dass die nationalsozialistische Machtübernahme - wie die Besetzung des Gewerkschaftshauses in München im März 1933 - um ein Haar hätte verhindert werden können, wenn jemand den Mut gehabt hätte zu ,schreien', sich zu widersetzen, zu protestieren. Aber niemand stieß diesen erlösenden Schrei aus. Es ist wahrscheinlich, dass dies aus seiner Perspektive im Jahre 1933 so ,harmlos` ausgesehen hat, er war neunzehn und hatte keinen Überblick. Doch die Perspektive der Jahre 1950, 1951 muss ihm Einblick in die politischen Konstellationen der späten Weimarer Republik gegeben haben. Er hat wissen müssen, dass es Menschen gab, die protestierten und ,schrien“ und den Nationalsozialismus nicht aufhalten konnten, weil seine Ideologie doch die Unterstützung der Mehrheit bekam.

Es scheint, dass sich Andersch bereits früh, unmittelbar nach seiner Flucht zu den Amerikanern, einen Habitus zurechtlegte, den die meisten jungen Deutschen in 
der eigenen Vorstellung vertraten. Dieser Habitus zeigt eine - um mit Bourdieu zu sprechen - kollektive Disposition zum Gehorsam, doch auch individuelle Veranlagung zur kritischen Reflexion, ein intellektuelles ,Naturell', das den Durchschnittsmenschen auch in der Armee den Anstand bewahren ließ. Inwieweit dieses Bild, das Andersch in seinen Werken entwickelt, eine Entsprechung in der damaligen Wirklichkeit hatte, ist für einen Historiker von grundsätzlicher, für eine Literaturwissenschaftlerin von sekundärer Bedeutung.

\section{3.}

Die Kirschen der Freiheit, Anderschs erster Erfolg, entwickelte sich aus früheren literarischen Versuchen, die bereits gegen Mitte der 40er Jahre junge Deutsche (deutsche Soldaten) deutlich als zweifelnde, reflektierende, gleichzeitig auch verirrte Menschen präsentierten, die durchaus von ihrer Beschaffenheit her die Bereitschaft zur Wandlung - oder, wie die Alliierten dies nannten, zur Reeducation - zeigten.

Der bekannteste Text, der für Die Kirschen der Freiheit als Vorlage diente, ist die Erzählung Flucht in Etrurien, die zum ersten Mal im August 1950 in der F.A.Z. als Fortsetzungserzählung erschien. Die Autobiographie integriert darüber hinaus manche Episoden aus der ,Reportage“ Amerikaner - Erster Eindruck, einer Skizze, die wahrscheinlich im Kriegsgefangenenlager Fort Kearney entstand.

Flucht in Etrurien erzählt den gleichen Plot wie das Kapitel Fahnenflucht in Die Kirschen der Freiheit: der Held -, in der Erzählung heißt er Werner, in der Autobiographie ist es eine Ich-Figur, die man mit Alfred Andersch identifizieren kann - ist deutscher Soldat und befindet sich im Frühsommer 1944 mit seiner Einheit in Italien, nordwestlich von Rom, in der Nähe von Vejano. Die Soldaten sollen an die Front versetzt werden, doch alle anderen Einheiten, denen sie begegnen, sind im Rückzug begriffen. Der Held plant die Flucht von seiner Truppe, die ihm relativ leicht gelingt. Beide Werke enden mit der Szene, in der der Held im Gras unter einem Kirschbaum liegt und seine Freiheit genießt. In der autobiographisch erzählten Geschichte flieht der Held (Andersch) allein. Der Kamerad, der mit ihm zurückbleibt und ihm hilft, einen Radreifen zu flicken, will unbedingt zu der Einheit zurück und lässt Andersch, der versichert, er komme bald nach, allein. An diesen Kameraden konnte sich der Autor nach einigen Jahren nur schwer erinnern: „Eine blasse Figur, dieser Werner, in meinem Buch und im Leben. Ich entsinne mich seiner nur noch schwach“ (Andersch, 1968, S. 35). Immerhin gab Andersch dem Helden seiner Erzählung Flucht in Etrurien ${ }^{2}$ den Namen Werner, aus Zufall oder doch in der Absicht, diesen zufälligen Gefährten der letzten Tage seines Soldatenlebens zu ver-

\footnotetext{
${ }^{2}$ Erstausgabe in Frankfurter Allgemeine Zeitung als Fortsetzungsroman zwischen dem 10. und 22. August 1950.
} 
ewigen. Dies hat insofern eine Bedeutung, als in der frühen Erzählung die Handlung zu einer kollektiven Flucht ausgebaut wurde. Der Protagonist überredet Erich, einen Kameraden, den er vor dem letzten Einsatz retten will, mit ihm die Truppe zu verlassen. Sie haben einen Gehilfen, Unteroffizier Alex, der selbst aus Pflichtbewusstsein und Solidarität bei seinen Leuten bleiben will, doch die anderen zwei laufen lässt. Erich schwankt lange, weil er zu Hause eine Braut hat, die von ihm erwartet, dass er für das Vaterland kämpft. Werner und Erich fliehen gemeinsam (mit Erlaubnis und Segen Alexanders, des Unteroffiziers!). In der Nacht wird Erich von einer Giftschlange gebissen und stirbt trotz der Hilfe italienischer Bauern. Werner rettet sich und isst in der oben genannten Schlussszene ,die Kirschen der Freiheit ${ }^{*}$. Die beschriebene Situation ist zwar wahrscheinlich, doch - so würden Historiker urteilen - unglaubwürdig. Der Unteroffizier Alex sagt zu dem ihm unterstellten Soldaten: „Du weißt, daß ich nicht dazu gehöre. Aber ich muß bei ihnen bleiben. Ein paar müssen doch bei ihnen bleiben“" (Andersch, 1981, S. 111), und er lässt die beiden frei, sie trennen sich von der Truppe und werden auch von anderen Menschen, die sie unterwegs treffen, nicht denunziert, nicht einmal von einem deutschen Feldwebel, der sich selbst offensichtlich auf der Flucht befindet:

\footnotetext{
Aber es seien doch noch genügend deutsche Truppen vorne, meinte Werner. Ob er ihre Einheit gesehen habe?

„Radfahrer“ fragte der Feldwebel und sah prüfend auf ihre Räder. Ja, so ein paar arme Irre habe er da vorne (...) gesehen. Das seien aber beinahe die einzigen, die noch vorne am Feind wären.

„Beeilt euch man“, grinste er, „,damit ihr dabei seid, wenn euer Haufen vereinnahmt wird!“ (Andersch, 1981, S. 147-148)
}

In Die Kirschen der Freiheit verzichtet Andersch auf solche Episoden. Er lässt seinen Helden eine eigene Entscheidung treffen, die von anderen Soldaten nicht gebilligt, geschweige denn geteilt wird. Handelt der Held in Flucht in Etrurien aus einem Gemeinschaftsgeist heraus, wird in Die Kirschen der Freiheit die Opposition Anderschs - der sich selbst als reflektierender und kritischer junger Mann zeigt - zu seinen Kameraden hervorgehoben:

Sie hingen mir meterlang zum Hals heraus, die sogenannten Kameraden. Sie kotzten mich regelrecht an. Das Schlimmste an ihnen war, daß sie immer da waren. Kameradschaft - das bedeutete, daß man niemals allein war. Kameradschaft hieß, daß man niemals eine Tür hinter sich zumachen und allein sein konnte. (Andersch, 1968, S. 63)

Solche Formulierungen lesen sich wie eine Polemik gegen den Geist, den sowohl Remarque als auch paradoxerweise Jünger in der Zeit der Weimarer Republik in Erinnerung an den Ersten Weltkrieg populär machten: Unabhängig davon, ob in pazifistischer oder national-militaristischer Einkleidung, wurde das Zusammengehörigkeitsgefühl, ein männlicher Gemeinschaftsgeist glorifiziert. Der Ich-Erzähler in Die Kirschen der Freiheit fragt dagegen unverblümt: „Ihretwegen etwa sollte ich 
nicht desertieren? Aus ,Kameradschaft' sollte ich ,beim Haufen bleiben?““ (Andersch, 1968, S. 67). Doch es befallen ihn manchmal Zweifel. Er überlegt, ob er nicht einen von den Kameraden überzeugen sollte, sich der Flucht anzuschließen - dies tat der fiktive Held Werner in Flucht in Etrurien. Wenn man nach über sechzig Jahren die beiden Werke miteinander vergleicht, fällt auf, dass Andersch die unwahrscheinliche Unterstützung der Flucht seitens der Kameraden zwar auf der Ebene der Handlung korrigiert, doch nicht ganz aufgeben will. Er versucht dem Einsatz der deutschen Soldaten im Zweiten Weltkrieg eine Deutung zu geben, die im Einzelfall (in seinem Fall?) möglicherweise der Wahrheit entsprach, doch sich in sehr vielen Fällen dem historischen Wissen über diese Zeit entzieht:

(...) Millionen ziemlich tapferer Männer, die es im Bauch hatten, daß es im Grunde Quatsch war, zu kämpfen. Wenn sie's taten - und oft taten sie es gut -, dann unter Zwang oder um gerade noch eben das Gesicht zu wahren, weil man das Gesicht wahren mußte, als die Vollidioten bei den anderen gesiegt hatten und mit der Formel von der ,bedingungslosen Übergabe‘ (...) anrückten. Die deutschen Soldaten haben das Gesicht gewahrt, aber es hat im letzten Kriege niemals eine ,Wehrmacht' gegeben, sondern einzig und allein Millionen bewaffneter Männer, deren größter Teil nicht die geringste Lust hatte, zu kämpfen. (Andersch, 1968, S. 80)

Ohne die Authentizität von Anderschs eigenen Ansichten hinterfragen zu wollen, muss man doch sein Urteil über die Haltung der deutschen Soldaten als eine Wunschprojektion deuten, die der Wirklichkeit wenig entsprach. So steckt auch sein Bericht voller Widersprüche. An einer anderen Stelle versucht er zu erklären, warum seine Kameraden doch nicht - wie er selbst - desertierten:

Es war einfach das „Beim-Haufen-Bleiben“ selbst, der Herdeninstinkt, mit Terror und Propaganda unablässig in sie hineingetrommelt (...). Man war schon fast selbständig, wenn man dem Trieb zum einfachen Aufgehen im Massenschicksal ein paar unklare Vorstellungen von Kameradschaft und Verteidigung des Vaterlandes beimischte oder einfach von Natur aus kampfeslustig war. Die meisten deutschen Soldaten bewegten sich in diesem Kriege nicht wie Träumer, auch nicht wie Betrunkene, sondern wie Gebannte; wer unter der Gewalt des bösen Blickes steht, sieht nicht mehr Iris und Pupille des Hypnotiseurs. Sein Bewußtsein ist ausgeschaltet, er fühlt nur noch den Bann. (Andersch, 1968, S. 100-101)

Andersch gibt also doch zu, dass die Soldaten der nationalsozialistischen Propaganda erlegen waren und nicht selbständig urteilen konnten. Der „Bann“ des „,bösen Blicks" erinnert an die viel später formulierten Thesen von Historikern, die man als ,Intentionalisten' bezeichnet, und die die Ursache der nationalsozialistischen Verbrechen vor allem auf die psychopathologische Veranlagung Hitlers zurückführten. ${ }^{3}$ So konnte man in der Folge einer solchen Deutung den Durchschnittsmenschen teilweise oder voll entlasten. Andersch war nicht der einzige Autor, der nach dem

\footnotetext{
${ }^{3}$ Der Kontroverse zwischen den ,Intentionalisten` und den ,Funktionalisten` oder ,Strukturalisten“ wurden sehr viele Publikationen gewidmet. Von den neueren ist der Artikel von Wehler, 2007 zu empfehlen.
} 
Zweiten Weltkrieg versuchte, die persönliche Schuld des einfachen Soldaten in Frage zu stellen. Auch Martin Walser solidarisierte sich sowohl in der linken als auch in der ,nationalen' Phase seines Schaffens mit dem ,kleinen Mann', allerdings in viel spektakuläreren Formulierungen, etwa - aus dem Jahre 1987, also 35 Jahre nach Anderschs Die Kirschen der Freiheit: „Es war ein Krieg, der mag so ungerecht angefangen worden sein wie auch immer: das hat aber nicht der Soldat aus Wasserburg zu verantworten. Den spreche ich frei““ (Frank, N. \& Köhler, J., 1987, S. 222).

Andersch korrigierte immerhin seine frühere übertrieben optimistische und unglaubwürdige Version aus Flucht in Etrurien, darüber hinaus betonte er die Befangenheit seiner Erinnerungen: „Dieses Buch will nichts als die Wahrheit sagen, eine ganz private und subjektive Wahrheit. Aber ich bin überzeugt, daß jede private und subjektive Wahrheit, wenn sie nur wirklich wahr ist, zur Erkenntnis der objektiven Wahrheit beiträgt“" (Andersch, 1968, S. 71).

In der Autobiographie versucht der Autor nicht, seine Desertion als Beweis einer besonders mutigen Haltung zu erklären. Er betont mehrmals, dass er frei werden wollte und eine Gelegenheit suchte, sich von der Truppe zu entfernen; er habe Angst gehabt, sowohl vor dem sinnlosen Tod an der Front als auch vor der Strafe, die ihm drohte, wäre er in die Hände der Feldpolizei geraten (Reinhardt, 1990, S. 91-104, bes. S. 102: Kap. 6: , Uns trägt das Ungesungene - Desertion). Max Frisch betonte andererseits 1979 in seiner Laudatio auf Andersch die besondere Bedeutung der Desertion, die eine Geste nationaler Emanzipation darstelle, ,der Selbstbestimmung, was ein lebensgefährlicher Akt" gewesen sei (Frisch, 2014, S. 110). Ob hier eine antifaschistische, gar oppositionelle Gesinnung für Andersch eine Rolle spielte, Erinnerung an sein jugendliches Engagement für den Kommunismus oder einfach das Bedürfnis nach Rettung angesichts des sichtbar verlorenen Krieges, lässt sich heute nicht mehr rekonstruieren. Charakteristisch ist, was Frisch in einer Notiz über Andersch erzählte, die in sein Tagebuch 1966-1971 aufgenommen werden sollte, was freilich von Andersch schroff abgelehnt wurde und zum einige Jahre dauernden Zerwürfnis zwischen den beiden Freunden und Nachbarn führte:

Einmal fragte ich ihn nach seinen Erlebnissen; ziemlich betreten verwies er auf seine Prosa, die ich selbstverständlich kenne, Kirschen der Freiheit, auch andere Texte, die Auskunft geben. Ich meinte in diesem Augenblick aber nicht den Schriftsteller Alfred Andersch, sondern ihn privat. Das war für ihn (...) eine ungehörige Frage. (Frisch, 2014, S. 85)

Die Erinnerung von Frisch hätte ein falsches Bild von Andersch vermitteln können - davon könnte Anderschs damalige Entrüstung über die Tagebuch-Notiz zeugen. Die Weigerung, über Kriegserlebnisse zu sprechen, lässt vermuten - was Frisch andeutete -, dass Andersch die literarische Fiktion der eigenen Werke für sein Leben hielt. Nicht von der Hand zu weisen war der Verdacht, dass sich der deutsche Kollege bewusst eine Lebensgeschichte schreibend zurechtlegte. 
Anderschs Biograph, Stephan Reinhardt, macht auf einen Brief an die Mutter aufmerksam, den Andersch vor seinem Fronteinsatz schrieb:

So freue ich mich jetzt, daß ich gesund bin und nicht dem inneren Schweinehund nachzugeben brauche oder kann. Dabei huldige ich keinem flachen Optimismus (...). Aber es gibt einen ganz untrüglichen Instinkt für die Pflicht und die Verantwortung und ein Gefühl, wo die wahren Werte des Lebens sitzen, und der gibt einem das Recht auf Fröhlichkeit. (Andersch 1986, S. 15-16)

Reinhardt (1990) nennt diesen Brief bizarr, diplomatisch und doppeldeutig (S. 88). Es bleibt unbeantwortet, ob die Doppeldeutigkeit vorsätzlich war, ein Schutz gegen die mögliche Zensur, oder ob der junge Autor wirklich „Pflicht“ und „Verantwortung“ als Quelle der „Fröhlichkeit“ empfand, wie man dies verstehen kann. Andere Briefe an die Mutter, die er aus Italien schrieb, zeugen durchaus von einer gewissen Selbstzufriedenheit, ja Freude, dass das Schicksal Andersch nicht anderswohin, sondern nach Italien verschlug, wo er trotz der Mühen des Militärlebens sowohl die Landschaft als auch manch ein Glas Chianti genießen konnte. Es scheint, dass die einstige linke Gesinnung, Solidarität für Unterdrückte und Verfolgte als Gründe für die Desertion keine große Rolle spielten. In einem anderen autobiographischen Text, Amerikaner - Erster Eindruck, drückt er Bewunderung für die demokratischen Strukturen der amerikanischen Armee aus, gleichzeitig verteidigt er die deutschen Soldaten, die vor der schweren Entscheidung standen, den Kameraden (nicht unbedingt Hitler!?) treu zu bleiben oder Hochverrat zu begehen: „(...) die vielen kleinen Leute, die glauben, einen nationalen Krieg unterstützen zu müssen“" (Andersch, 2004a, S. 319).

\section{4.}

Anderschs Selbstbild schreibt sich in die Wunschprojektion von seiner Generation ein, die er als von Deutschen (deutschen Männern), die nicht unmittelbar in den NS-Machtapparat verstrickt waren, jedoch an den Kriegshandlungen teilnahmen, geprägt darzustellen versucht. Ohne den Begriff zu nennen, widerspricht er der These von der kollektiven Schuld, vielmehr attestiert er seinen Kameraden und den einfachen deutschen Bürgern eine kollektive ,Unschuld‘, die er nicht beweisen kann. Er kann sie aber ,erschreiben', indem er seine eigenen Erlebnisse und Emotionen literarisch der kollektiven, Wir'-Form zuordnet.

\section{Bibliographie}

Andersch, A. \& Acklin, J. (2002). Alfred Andersch im Gespräch mit Jürg Acklin v. 19., 20. Januar 1980. In W. Stephan (Hrsg.), Materialen zu, Die Kirschen der Freiheit 'von Alfred Andersch. Zu einem Buch und zu seiner Geschichte (S. 198-202). Zürich: Diogenes. 
Andersch, A. (2004a). Amerikaner - Erster Eindruck. In D. Lamping (Hrsg.), Andersch, A. Erzählungen. Autobiographische Berichte. Gesammelte Werke in zehn Bänden. Band 5 (S. 305-322). Zürich: Diogenes.

Andersch, A. (2004b). Der Seesack. In D. Lamping (Hrsg.), Andersch, A. Erzählungen. Autobiographische Berichte. Gesammelte Werke in zehn Bänden. Band 5 (S. 415-439). Zürich: Diogenes.

Andersch, A. (1968). Die Kirschen der Freiheit. Ein Bericht. Zürich: Diogenes (Originalwerk erschienen 1952).

Andersch, A. (1986). , ...einmal wirklich leben. 'Ein Tagebuch in Briefen an Hedwig Andersch 1943-1975. Herausgegeben von W. Stephan. Zürich: Diogenes 1986.

Andersch, A. (1981). Flucht in Etrurien. Zwei Erzählungen und ein Bericht. Zürich: Diogenes.

Andersch, A. (1969). Hohe Breitengrade oder Nachrichten von der Grenze. Zürich: Diogenes.

Andersch, A. (1972). Norden Süden rechts und links: von Reisen und Büchern 1951-1971. Zürich: Diogenes.

Bargelli, R. (2008). Die deutsche Nachkriegsliteratur und der italienische Neorealismus. Alfred Anderschs Reisebericht „Aus einem römischen Winter“. In R. G. Czapla \& A. Fattori (Hrsg.), Die verewigte Stadt. Rom in der deutschsprachigen Literatur nach 1945 (S. 152-162). Bern: Lang.

Böll, H. (1992). Wo sind die Deserteure? In W. Stephan (Hrsg.), Über ,Die Kirschen der Freiheit ‘ von Alfred Andersch (S. 132-135). Frankfurt a.M.: Frankfurter Verlagsanstalt.

Döring, J. (2011). Zur Textgenese von Anderschs Kirschen der Freiheit. Eine Autopsie ausgewählter Passagen des handschriftlichen Befundes. In J. Döring \& M. Joch (Hrsg.), Alfred Andersch revisited: werkbiographische Studien im Zeichen der Sebald-Debatte (S. 13-47). Berlin \& Boston: De Gruyter.

Frank, N. \& Köhler J. (1987). „Ich hab’ so ein Stuttgart-Leipzig-Gefühl.“ Ein Gespräch mit Martin Walser. Stern, 12, 220-224.

Frisch, M. (2014). Laudatio auf Alfred Andersch. In J. Bürger (Hrsg.), Alfred Andersch. Max Frisch. Briefwechsel (S.108-116). Zürich: Diogenes.

Holthusen, H. E. (1992). Reflexionen eines Desertuers. In W. Stephan (Hrsg.), Materialen zu ,Die Kirschen der Freiheit' von Alfred Andersch. Zu einem Buch und zu seiner Geschichte (S. 118-127). Frankfurt a.M.: Frankfurter Verlagsanstalt.

Jabłkowska, J. (2016). „Diese Unruhe ist es, die in den geglückten Reisebüchern tickt.“ Alfred Anderschs Italienbild. In N. Ächtler (Hrsg.), Alfred Andersch. Engagierte Autorschaft im Literatursystem der Bundesrepublik (S. 148-162). Stuttgart: Metzler.

Kunz, E. (1994). Reise- und Landschaftsprosa seit 1944. In I. Heidelberger-Leonard \& V. Wehdeking (Hrsg.), Alfred Andersch. Perspektiven zu Leben und Werk (S. 65-74). Opladen: Westdeutscher Verlag.

Lamping, D. (2000). Erzählen als Sinn-Suche. Formen und Funktionen autobiographischen Erzählens im Werk Alfred Anderschs. In R. Zymner (Hrsg.), Erzählte Welt - Welt des Erzählens. Festschrift für Dietrich Weber (S. 217-229). Köln: Edition chora.

Lejeune, Ph. (1994). Der autobiographische Pakt. (W. Bayer \& D. Hornig Übers.). Frankfurt a.M.: Suhrkamp.

Reinhardt, S. (1990). Alfred Andersch. Eine Biographie. Zürich: Diogenes.

Scherpe, K. R. (2015). Literatur, das Politische betreffend. Alfred Andersch. Weimarer Beiträge, 61 (3), 437-453.

Sebald, W. G. (1993). Between the devil and the deep blue sea. Alfred Andersch. Das Verschwinden in der Vorsehung. Lettre International, 20, 80-84.

Sebald, W. G. (1999). Luftkrieg und Literatur: Mit einem Essay zu Alfred Andersch. München: Hanser.

Seubert, R. (2011). „Mein lumpiges Vierteljahr Haft ...“. Alfred Anderschs KZ-Haft und die ersten Morde von Dachau. Versuch einer historiographischen Rekonstruktion. In J. Döring \& M. Joch 
(Hrsg.), Alfred Andersch revisited: werkbiographische Studien im Zeichen der Sebald-Debatte (S. 47-146). Berlin: De Gruyter.

Stephan, W. (1992) (Hrsg.). Materialen zu ,Die Kirschen der Freiheit'von Alfred Andersch. Zu einem Buch und zu seiner Geschichte. Frankfurt a.M.: Frankfurter Verlagsanstalt.

Tuchel, J. (2008). Alfred Andersch im Nationalsozialismus. In M. Korolnik \& A. Andersch-Korolnik (Hrsg.), Sansibar ist überall. Alfred Andersch. Seine Welt - in Texten, Bildern, Dokumenten (S. 31-41). München: text und kritik.

Tuchel, J. (2011). Respondenz zum Beitrag von Rolf Seubert: „Mein lumpiges Vierteljahr Haft ...“. In J. Döring \& M. Joch (Hrsg.), Alfred Andersch revisited: werkbiographische Studien im Zeichen der Sebald-Debatte (S. 147-152). Berlin: De Gruyter.

Wehler, H. U. (2007). Intentionalisten, Strukturalisten und das Theoriedefizit der Zeitgeschichte. In N. Frei (Hrsg.), Martin Broszat, der ,Staat Hitlers ' und die Historisierung des Nationalsozialismus (S. 71-76). Göttingen: Wallstein. 